$\mathrm{RM}-75-26$

A REDUCED DIMENSIONALITY METHOD FOR

THE STEADY-STATE KALMAN FILTER

J. Casti

June 1975

Research Memoranda are informal publications relating to ongoing or projected areas of research at IIASA. The views expressed are those of the author, and do not necessarily reflect those of IIASA. 


\section{A Reduced Dimensionality Method for the Steady-State Kalman Filter}

J. Casti

\section{Introduction}

A venerable problem in the modern system theory literature is the so-called Kalman filtering question in which we desire to determine the optimal least-squares estimate of a noisy signal, given a model for the observation process (details later). The central importance of this problem is primarily due to the fact that its solution is recursive, enabling one to, in principle, employ straightforward computational methods for the solution. However, if the dimension of the system state is large, the computational problem is no longer negligible since, in the Kalman formulation, to determine the optimal filter gain we must solve $O\left(n^{2}\right)$ simultaneous quadratic equations, where $\mathrm{n}$ is the dimension of the state. In these cases, many refinements of the standard algorithms have been proposed to take advantage of the structure which the system presents, e.g. sparseness, weak coupling, and so forth.

In recent years, mathematical steps have been taken to substantiate the conjecture that, regardless of the dimension of the system state, if the dimensions of the input and output spaces are small, it should be possible to exploit this situation and reduce the computational complexity of the equations needed to calculate the optimal filter gain 
(or the optimal feedback control law). Beginning with the work [7], and continued in $[4,6,10]$, it has been shown that this conjecture is valid and that indeed it is possible to reduce the number of needed equations to $O(n)$ without increasing the analytic complexity of the problem (still quadratically nonlinear). The ideas which motivated the new filtering equations had their origin in radiative transfer, special cases having been developed by Ambartsumian [1] and Chandrasekhar [9] in the 1940's. As a consequence of the transport terminology, the new filtering functions have been called "generalized $\mathrm{X}-\mathrm{Y}$ functions" [4] or "Chandrasekhar-type" algorithms [10]. Unfortunately, the finite interval versions of the $\mathrm{X}-\mathrm{Y}$ functions do not readily lend themselves to an algebraic equation for the infinite interval case, in contrast to the situation for standard matrix Riccati equation of optimal filtering. Thus, our objective in this paper is two-fold: to review the basic hypotheses and results of the finite-interval reduction for the Kalman filtering problem and secondly, to present a derivation of the appropriate algebraic equation which may be used instead of the algebraic Riccati equation in order to determine the optimal steady-state gain function. In addition, we present results of comparative numerical experiments, as well as some directions for future work. 


\section{The Kalman Filter}

The standard Kalman filtering problem which we study is the following: a noisy signal $\mathrm{z}$,

$$
z(t)=H x(t)+v(t), \quad 0 \leq t<\infty
$$

is observed. We assume $\mathrm{z}$ to be the output of the linear system

$$
\frac{d x}{d t}=F x+G u, \quad x(0)=x_{O},
$$

where $u$ is a white noise process. Here $x, u, z$ are $n, m$, and p-dimensional vectors, respectively, with F, G, H being constant matrices (we are only interested in the stationary case in this paper). The observation noise $v$, the system noise $u$, and the initial state $x_{O}$ are assumed to satisfy the statistical conditions

$$
\begin{aligned}
& E[v(t)]=E[u(t)]=E\left[x_{O}\right]=0, \\
& E\left[v(t) v^{\prime}(s)\right]=I \delta(t-s), \\
& E\left[u(t) u^{\prime}(s)\right]=Q \delta(t-s), \\
& E\left[v(t) u^{\prime}(s)\right]=0, \\
& E\left[x_{O} x_{O}^{\prime}\right]=\Gamma, \quad 0 \leq t<s<\infty .
\end{aligned}
$$

Further, the covariance matrices $Q$ and $\Gamma$ satisfy the definiteness properties 


$$
Q \geq 0, \quad \Gamma \geq 0
$$

Thus, the noises in the system are independent, zero-mean white gaussian processes.

Within the context of the foregoing situation, we desire to determine an estimate, $\hat{x}(t)$, of $x$ such that for every constant vector $\lambda$ we have

$$
E[\lambda \cdot(x(t)-\hat{x}(t))]^{2} \leq E[\lambda \cdot(x(t)-f(z(t)))]^{2}
$$

for every measurable functional $f(z(t))$ such that

$$
E[\hat{x}(t)]=E[x(t)]=E[f(z(t))]=0
$$

In other words we wish to choose $\hat{x}(t)$ to minimize the covariance of the error between the true state $x$ and the estimated state $\hat{x}$.

The classical Kalman filtering solution to the foregoing problem shows that the optimal estimate $\hat{x}(t)$ is generated by the differential equation

$$
\begin{aligned}
& \frac{\hat{d x}}{d t}=\hat{F x}(t)+P(t) H \cdot[z(t)-\hat{H x}(t)], \\
& \hat{x}(0)=0,
\end{aligned}
$$

where $P(t)$ is the solution of the matrix Riccati equation 


$$
\begin{aligned}
& \frac{d P}{d t}=G Q G^{\prime}+F P+P F^{\prime}-P H^{\prime} H P, \\
& P(O)=\Gamma .
\end{aligned}
$$

For future reference, we note that the kalman gain function $K(t)$ is given by

$$
K(t)=P(t) H^{\prime} \text {. }
$$

The steady-state gain $K(\infty)$ is obtained by solving the algebraic Riccati equation

$$
\mathrm{GQG}+\mathrm{FP}(\infty)+\mathrm{P}(\infty) \mathrm{F}^{\prime}-\mathrm{P}(\infty) \mathrm{H}^{\prime} \mathrm{HP}(\infty)=0,
$$

or

$$
\mathrm{GQG}+\mathrm{FP}(\infty)+\mathrm{P}(\infty) \mathrm{F}^{\prime}-\mathrm{K}(\infty) \mathrm{K}^{\prime}(\infty)=0
$$

Our subsequent development will concentrate on deriving a substitute for Eq. (6) involving only np unknown quantities, rather than the $\mathrm{n}(\mathrm{n}+1) / 2$ unknowns in (6) (recall that $\mathrm{p}$ is the dimension of the observation process (1)). 


\section{Generalized X-Y Functions}

Since the finite interval version of the generalized $\mathrm{X}-\mathrm{Y}$ functions for the Kalman filter are of some interest in their own right, we present them without proof in this section. The results follow the development in [4]. The basic result is

\section{Theorem 1. $[4,10]$ Let}

(i) $\operatorname{rank} \mathrm{Z}\left(=\mathrm{GQG}+\mathrm{F} \Gamma+\Gamma \mathrm{F}^{\prime}-\Gamma \mathrm{H}^{\prime} \mathrm{H} \Gamma\right)=\mathrm{r}$,

(ii) rank $\mathrm{H}^{\prime} \mathrm{H}=\mathrm{p}$,

and let $\mathrm{Z}$ be factored as $\mathrm{Z}=\mathrm{BB}^{\prime}$, where $\mathrm{B}$ is an $\mathrm{n} \times \mathrm{r}$ matrix. Then the solution to the matrix Riccati equation (4) satisfies the algebraic relation

$$
F P(t)+P(t) F^{\prime}=L(t) L^{\prime}(t)-K(t) K^{\prime}(t)-G Q G^{\prime}, 0 \leq t \leq \infty
$$

where $L$ and $K$ are $n \times r, n x p$ matrix functions, respectively, satisfying the equations

$$
\begin{aligned}
& \frac{d L}{d t}=(F-K(t) H) L, L(O)=B, \\
& \frac{d K}{d t}=L(t) L^{\prime}(t) H^{\prime}, \\
& K(O)=\Gamma H^{\prime},
\end{aligned}
$$

\section{$\underline{\text { Remarks }}$}

1) Eqs. (7)-(8) represent a system of $n(p+r)$ equations suitable for computing the functions $L$ and $K$. The importance of this representation is that, by definition, $K(t)=P(t) H^{\prime}$, 
the Kalman gain itself. Thus, not only are there fewer equations in the L-K system, but they allow a direct computation of the physically relevant quantity $K$.

2) The algebraic relation (7) is often useful in determining the error covariance $P(t)$ for selected values of $t$, provided that the inversion of (IQF $+F \otimes I)$ can be readily accomplished (here denotes the Kronecker product).

3) Since $(8)-(9)$ represent $n(p+r)$ equations, a computational savings over the Riccati equation (4) is anticipated whenever $p+r<(n+1) / 2$. However, from the rank conditions (i) and (ii), we see that $p$ is always equal to the dimension of observation process, while reis always bounded from above by the number of inputs to the system model. Thus, for most physical processes there is a high likelihood that the condition $p+r<(n+1) / 2$ will be satisfied.

\section{Steady-State Solutions}

In many instances, it is of interest to know the steady-state gain $K(\infty)$ so that either it may be used as a sub-optimal, easily implementable filter, or so that one can determine the minimal possible error covariance over an infinite observation horizon. In these cases the usual approach is to solve the algebraic Riccati equation (6) for its unique positive semi definite solution $P(\infty)$, then form the gain function $K(\infty)$. As earlier discussed, this procedure requires the solution of $n(n+1) / 2$ simultaneous quadratic 
equations. Our main result, given below, is to show that the optimal gain, $K(\infty)$, may be directly determined by solving np simultaneous quadratic equations. Thus, when $\mathrm{p}<(\mathrm{n}+1) / 2$, we have fewer equations. As will be pointed out below, our new equation also possesses some analytic features which may suggest its use even if $p>(n+1) / 2$.

The result upon which all else follows is the Steady-State Theorem: Assume F has no purely imaginary characteristic values and no real characteristic values symmetrically placed relative to the orgin. Then the steadystate gain $K$ satisfies the algebraic equation

$$
\sigma(K)=(H \otimes I)(I \otimes F+F Q I)^{-1} \sigma\left(K K^{\prime}-G Q G^{\prime}\right),
$$

where $\otimes$ denotes the Kronecker product and $\sigma$ is the column stacking operation, i.e. if $A=\left[\underline{a}_{\underline{i j}}\right]$ then

$$
\sigma(A)=\left(a_{11} a_{21} \cdots a_{n 1} a_{12} a_{22} \cdots a_{n 2} \cdots a_{n n}\right)^{\prime}
$$

Proof. Applying $\sigma$ to the algebraic Riccati equation (6), we have

$$
\sigma\left(\mathrm{FP}(\infty)+\mathrm{P}(\infty) \mathrm{F}^{\prime}\right)=\sigma\left(\mathrm{KK} \mathrm{K}^{\prime}-\mathrm{GQG} \mathrm{G}^{\prime}\right) \text {. }
$$

Now we utilize the following property of the operation $\sigma$ :

$$
\sigma(P A Q)=\left(Q^{\prime} \otimes P\right) \sigma(A),
$$


valid for all $\mathrm{P}, \mathrm{A}, \mathrm{Q}$ for which the product $\mathrm{PAQ}$ is defined. Applying this result to (11) yields

$$
\sigma(P(\infty))=(I \otimes F+F \otimes I)^{-1} \sigma\left(K K^{\prime}-G Q G^{\prime}\right) .
$$

But,

$$
\mathrm{K}(\infty)=\mathrm{P}(\infty) \mathrm{H}^{\prime},
$$

which implies

$$
\sigma(K)=(H \otimes I) \sigma(P(\infty)),
$$

which completes the proof.

There are several remarks in order regarding the above Theorem:

a) the presence of the matrix inverse $(I \otimes F+F \otimes I)^{-1}$ seems, at first glance, to present a serious computational obstacle, being an $\mathrm{n}^{2} \times \mathrm{n}^{2}$ matrix. However, being of special structure, it is possible to carry out this inversion utilizing the characteristic polynomial of $\mathrm{F}$ and inversion of an $\mathrm{n} x \mathrm{n}$ matrix (see [2] for details).

In addition, for many parametric studies the system dynamic matrix $F$ is reasonably well known and our interest is in exploring the effect of changing $\mathrm{G}, \mathrm{H}$, and/or $\mathrm{Q}$. Thus, the inversion need be done only once and stored for future use. 
b) the form of equation (10) is more well-suited to standard successive approximation algorithms than the usual algebraic Riccati equation since the unknown quantity appears by itself on the left side of the equation. While this is a minor point, it does enable us to directly appeal to many standard results from functional and numerical analysis in which a form

$$
\mathrm{u}=\mathrm{T}(\mathrm{u})+\mathrm{f}
$$

is assumed for the equation to be solved in the unknown $u$.

c) it is important to note that the number of equations in (10) depends only upon the number of observation points $p$ and is totally independent of the number $r$, which played a role in the finite-interval equations (8)-(9) for $L$ and $K$. In view of the proof of the Steady-State Theorem, one might conjecture that an equation for $\mathrm{K}$ alone could be developed for the finite-interval problem. Further remarks on this situation in the context of control theory, as well as implications of (10) for the Inverse Problem of Control are given in $[3,5,8,11]$. 


\section{Numerical Resuits}

Interesting as Eq. (10) may be on theoretical grounds, the proof is in the program and the final test is its numerical efficacy vis-à-vis the algebraic Riccati equation (6). To investigate this question, several comparative numerical experiments were performed involving the matrices

$$
Q=I \quad H=\left(\begin{array}{llll}
O & 0 & 0 & 1
\end{array}\right), \quad G=\left(\begin{array}{l}
1.7 \\
4.2 \\
0.85 \\
3.91
\end{array}\right),
$$

with six different $F$ matrices. In all cases $F$ has the companion form

$$
F=\left[\begin{array}{cccc}
0 & 1 & 0 & 0 \\
0 & 0 & 1 & 0 \\
0 & 0 & 0 & 1 \\
-a_{3} & -a_{2} & -a_{1} & -a_{0}
\end{array}\right] .
$$

Thus, $\mathrm{F}$ is completely determined by its characteristic roots. The computing procedure used was the following:

1) the finite-time Riccati equation (4) was integrated to a value $\mathrm{T}$ at which the relative change in its components is going from $T-\Delta$ to $T$ was less than $10^{-4}$. This determined an initial approximation $\mathrm{P}_{\mathrm{O}}$ to $\mathrm{P}(\infty)$. Using $\mathrm{P}_{\mathrm{O}}$ and a built in subroutine for solving sets of nonlinear algebraic equations 
(a hybrid procedure combining Newton iteration and steepest descent, see [11] for details and a program), the algebraic Riccati equation was solved to an accuracy in the residuals of $5 \times 10^{-5}$.

2) Using the initial guess $\mathrm{K}_{\mathrm{O}}=\mathrm{P}_{\mathrm{O}} \mathrm{H}^{\prime}$, the algebraic equation (10) was solved by the same algorithm as used in step (1) to the same degree of accuracy.

The results of the above experiments, calculated on the CDC Cyber 74 computer by Dr. O. Kirschner, are given in Table 1 .

Table 1. Computing times (in seconds) for four-dimensional problem.

\begin{tabular}{l|c|c|c}
$\begin{array}{l}\text { Case Number } \\
\text { (Roots of F) }\end{array}$ & Eq. (10) & Algebraic Riccati Eq. & $\begin{array}{c}\text { Approx. Time } \\
\text { Factor }\end{array}$ \\
\hline$-8,-8,-8,-8$ & 0.073 & 0.783 & 11 \\
$-15,-8,-8,-8$ & 0.102 & 0.706 & 7 \\
$-8,-1.4,1.5,9.5$ & 0.123 & 0.431 & 3.5 \\
$-5.5 \pm 3.5 i, 8.4,-12.4$ & 0.123 & 0.757 & 6 \\
$3.3,-7.5,-0.2 \pm 9.2 i$ & 0.119 & 0.818 & 7 \\
$-0.1 \pm i,-0.2 \pm 9.2 i$ & 0.087 & 0.575 & 7
\end{tabular}

From Table l, it is evident that Equation (10) results in a dramatic improvement over the computing time required for the algebraic Riccati equation. What is surprising, perhaps, is the large magnitude of the improvement since, on a strictly equation-counting basis, we would expect a factor of 
between two and three as there are four equations in (10) as opposed to ten equations in the algebraic Riccati equation (6). Thus, it is evident that not only does Eq. (10) represent fewer equations, but they exhibit better analytic behavior than the algebraic Riccati equation (6). This observation is consistent with other calculations carried out for the finite time equations (8) and (9) using a variable step integration routine. For a fixed accuracy, it was found that Eqs. (8)-(9) allowed a much larger integration step to be taken indicating a smoother right hand side than the Riccati equation (4). As yet, we have no analytic results to explain this phenomenon, but it seems most likely due to the fact that (8)-(9) separate the linear and quadratic parts of the Riccati equation into different equations, rather than combining them as is done in the Riccati equation.

\section{Discussion}

We have seen the possibility of substantially reducing the work needed to compute the steady-state gain $K(\infty)$ if the rank of the observation matrix is much less than the dimension of the state. Also, it has been seen that the actual computing time required is a function not only of the number of equations, but also depends upon the particular structure of the system. Thus, we pose the following issues as worthy of further study:

a) Numerical Studies: further experiments on many different system structures are needed to determine what 
structures of $F, G, H, Q$, give rise to particularly favorable (or unfavorable) computational cases vis-à-vis the algebraic Riccati equation. These experiments should also give limits as to what directions analytic investigations might profitably take.

b) Infinite-Dimensional Problems: some work has been done in extending the finite-time case to infinite-dimensional problems [8]. However, it remains to create the proper setting in order that the Steady-State Theorem be valid. 


\section{References}

[1] Ambartsumian, V. "Diffuse Reflection of Light by a Foggy Medium." Dokl. Akad. Nauk SSSR, 38 (1943), 229.

[2] Barnett, S., and Storey, C. Matrix Methods in Stability Theory, London, Nelson and Sons, Ltd., 1970.

[3] Casti, J. "A New Equation for the Linear Regulator Problem." J. Optim. Th. E Applic. (to appear October 1975).

[4] Casti, J. "Matrix Riccati Equations, Dimensionality Reduction and Generalized X-Y Functions." Utilitas Mathematica, 6 (1974), 95-110.

[5] Casti, J. "New Equations for the Time-Dependent Regulator Problem." IEEE Tran. Auto. Cont. (to appear August 1975).

[6] Casti, J. "Reduction of Dimensionality for Systems of Linear Two-Point Boundary Value Problems with Constant Coefficients." J. Math. Analy. Applic., 45 (1974), 522-531.

[7] Casti, J., Kalaba, R., and Murphy, K. "A New InitialValue Method for On-Line Filtering and Estimation." IEEE Trans. Info. Theory, IT-18 (1972), 515-518.

[8] Casti, J. and Ljung, I. "Some New Analytic and Computational Results for Operator Riccati Equations." SIAM Control J. (May 1975).

[9] Chandrasekhar, S. Radiative Transfer, New York, Dover Publ. Co., $196 \overline{0}$.

[10] Kailath, T. "Some New Algorithms for Recursive Linear Estimation in Constant Linear Systems." IEEE Tran. Info. Theory, IT-19 (1973), 750-760.

[11] Powell, M.J.D. "A Fortran Subroutine for Solving Systems of Nonlinear Algebraic Equations." AERE Report, Harwel1, 1972 . 